\title{
A New Method for Defocus and Astigmatism Correction in Electron Microscopy
}

\author{
M.E. Rudnaya*, W.van den Broek**, R.M.P. Doornbos***, S.C. Kho*, R.M.M. Mattheij*, J.M.L. Maubach* \\ * CASA, Eindhoven University of Technology, Den Dolech 2, 5612AZ, Eindhoven, The Netherlands \\ ** EMAT, University of Antwerp, Belgium \\ *** Embedded Systems Institute, Eindhoven, The Netherlands
}

Nowadays electron microscopy still requires an expert operator in order to manually obtain in-focus and astigmatism-free images. Both the defocus and the twofold astigmatism have to be adjusted regularly during the image recording process. Possible reasons are for instance the instabilities of the environment and the magnetic nature of some samples. For the next electron microscopy generations the manual operation has to be automated to improve the speed, the quality and the repeatability of the measurements.

A number of methods implemented on aberration-corrected microscopes are able to correct high and low aberrations, which include defocus and astigmatism. Unfortunately, these methods are not suitable for applications that require continuous operation since they are not fully automated (human operator has to point amorphous area or enter a range of parameters). Also, some of them make use of additional equipment, such as aberration correctors or a camera for Ronchigrams recording, which is not a part of every microscope.

We describe a new method for automated defocus and astigmatism correction in electron microscopy based on a sharpness function and derivative-free optimization [1]. The method

- corrects defocus and twofold astigmatism simultaneously;

- does not depend on image Fourier transform or Ronchigrams;

- does not require amorphous area or any other knowledge about the sample geometry;

- has been tested for simulations as well as for a real-world HAADF-STEM application.

The speed and accuracy of the method are comparable to those of an experienced human operator. Three examples of the method work are shown in Fig. 1-2. The method has been mainly tested for low-to medium magnifications. The application of the method to high resolution images could be the topic of the future research. Different optimization techniques can be employed for the method work: the Nelder-Mead simplex method and the Powell interpolation-based trust-region are discussed and compared for a real-world application running on a HAADF-STEM [2].

The current implementation of the method is based on the image variance sharpness function. Also, we could instead use a different image quality measure from a wide range of measures available in electron microscopy [3-4]. The method does not require any special hardware and is based on the general assumptions; therefore, it could be used for different types of microscopy (SEM, STEM, etc.). The method could be extended for correction of a wider range of microscope aberrations [5].

\section{References}

[1] M.E. Rudnaya et al., Ultramicroscopy, accepted 21 january 2011, in press (doi:10.1016/j.ultramic.2011.01.034).

[2] M.E. Rudnaya et al., Engineering Optimization. (2010), paper 01059.

[3] M.E. Rudnaya et al., Journal of Microscopy, 240 (2010) 38. 
[4] M.E. Rudnaya et al., Microsc. Microanal. 15 (Suppl. 2) (2009) 1008

[5] This work has been carried out as a part of the Condor project at FEI Company under the responsibilities of the Embedded Systems Institute (ESI). This project is partially supported by the Dutch Ministry of Economic Affairs under the BSIK program. We kindly acknowledge D.Langsweirdt (Technical University of Leuven), N.Venema (Technolution); S.Sluyterman, M.Otten (FEI), S. van Loo (ESI) for their help.

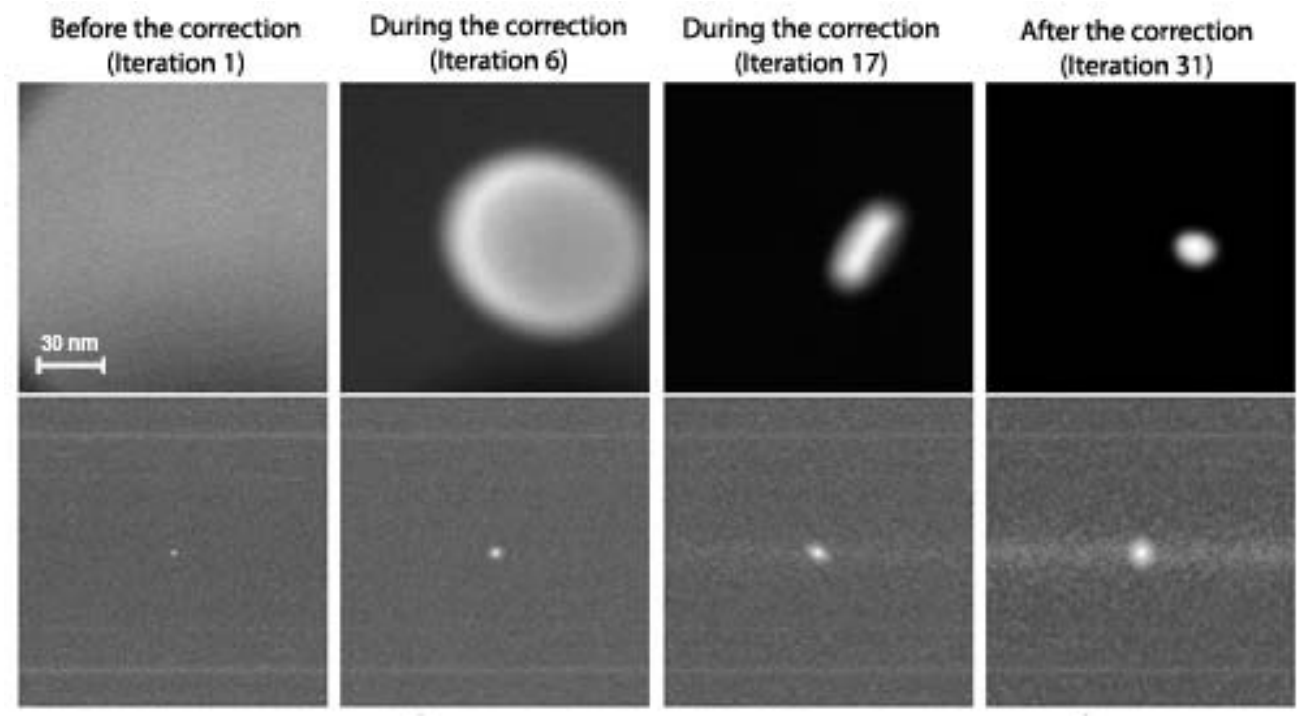

FIG. 1. Images of a gold particle and their Fourier transforms before the correction, during the correction and after the correction. On the left we see a particle, which is completely out-of-focus. On the right the image quality is the same as the one achieved by a human operator.
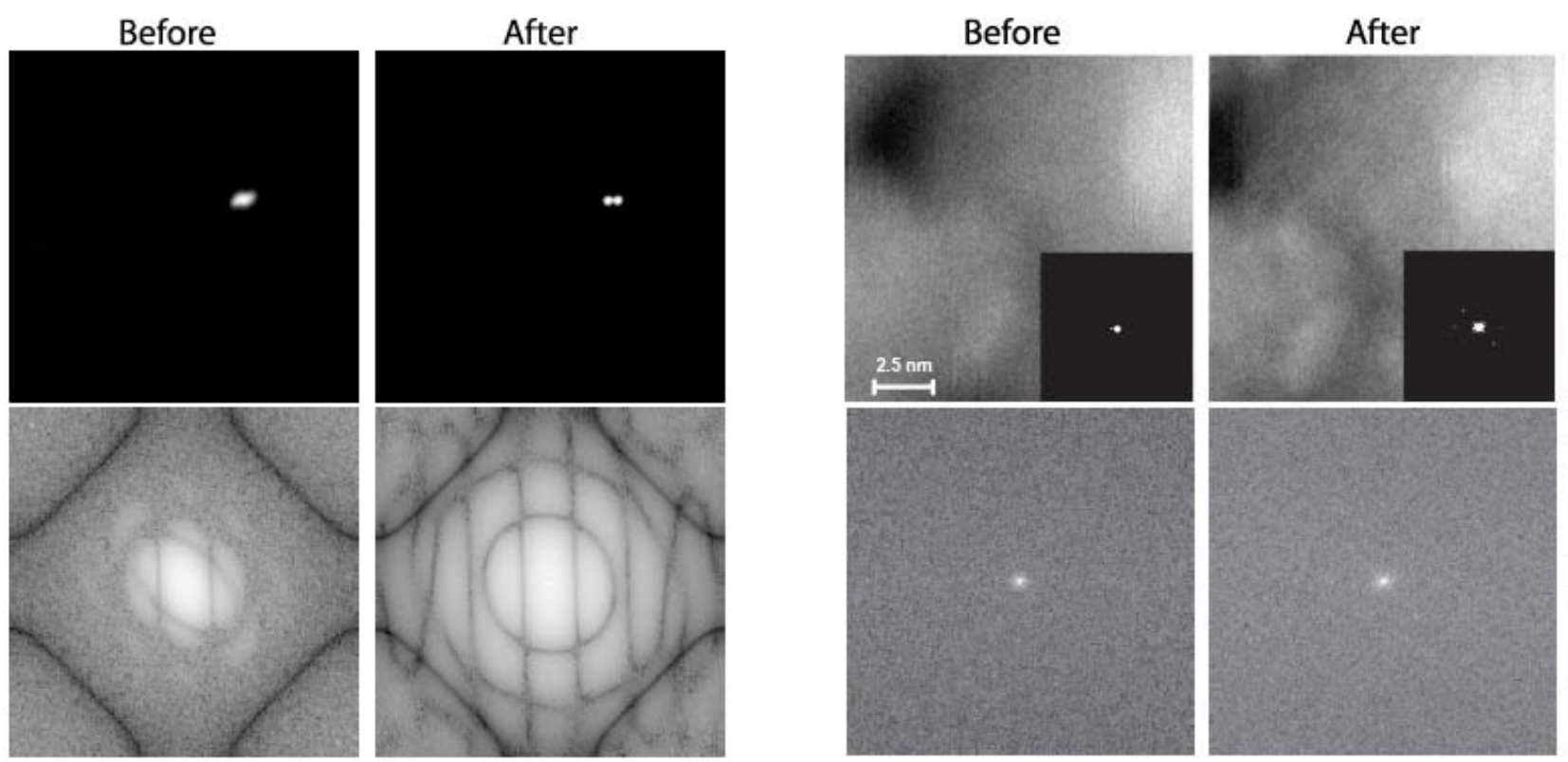

FIG. 2. From left to right: images of gold particles and their Fourier transforms, before and after the automated correction; images of carbon cross grating on the nanometer scale and their Fourier transforms before and after the automated correction. The alternative Fourier transform-based correction method could fail in these situations. 